\title{
Gangrena esplénica por Streptococcus anginosus post manga gástrica laparoscópica complicada con hemoperitoneo y comunicación gastroesplénica secundaria. Caso clínico
}

\author{
ANDRÉS ROJAS G., ${ }^{1, a}$, MARCELA OPAZO V., ${ }^{1, b}$, \\ PABLO MUÑOZ D. ${ }^{2}$, CARLOS CARVAJAL. ${ }^{3}$
}

\section{Splenic gangrene due to Streptococcus anginosus after a sleeve gastrectomy. Report of one case}

We report a 46-year-old woman, subjected to a laparoscopic sleeve gastrectomy, that had to be converted to open surgery due to the presence of adherences. She required an immediate new intervention due to a hemoperitoneum caused by a liver tear and venous bleeding from the splenic hilus. Both lesions were successfully repaired. In the postoperative period the patient had fever, leukocytosis and sialorrhea. A CAT scan showed a splenic infarction and a huge intra abdominal collection that communicated with the stomach. Streptococcus anginosus was isolated from the collection. The patient was managed with antimicrobials and percutaneous drainage with a favourable evolution and closure of the communication with the stomach.

(Rev Med Chile 2010; 138: 1539-1543).

Key words: Gastrectomy; Splenic infarction; Streptococcus anginosus.

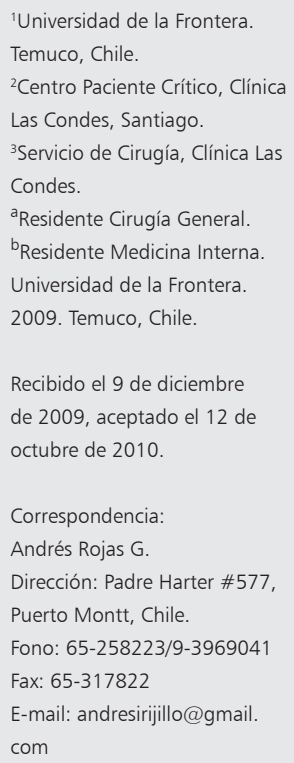

L a manga gástrica laparoscópica es una técnica en desarrollo durante la última década en el contexto de la cirugía bariátrica para el tratamiento de la obesidad mórbida. Hoy en día existen diversos estudios de cohorte prospectiva con resultados respecto a sus complicaciones a corto y largo plazo, las que pudiesen afectar su correcta implementación como alternativa de cirugía bariátrica.

Con respecto a esto último, de la literatura consultada mediante Medline ${ }^{\circledR}$, de los diversos estudios sobre complicaciones de esta cirugía, destaca la fuga de contenido gástrico como la más frecuente. Sin embargo, no econtramos casos de evolución tan tórpida y debido a un patógeno poco frecuente.

\section{Caso clínico}

Paciente de 46 años, mujer, obesa mórbida con índice masa corporal (IMC) 44 y antecedentes de dislipidemia, resistencia insulínica, apendicectomía, colecistectomía, reducción mamaria y banding gástrico previo fallido.

Ingresó para realización de manga gástrica laparoscópica que debió convertirse debido a adherencias gastrohepáticas y esplénicas intraperitoneales. Sin descripción de otras complicaciones tras término intervención. Durante post operatorio inmediato fue reintervenida por shock hipovolémico en contexto de hemoperitoneo secundario a desgarro hepático y sangrado venoso de hilio esplénico; ambos reparados exitosamente 
con taponamiento y clips respectivamente, decidiéndose así no instalar drenaje postoperatorio. Se indicó ceftriaxona y metronidazol profilácticos suspendidos a los 5 días debido a súbita eosinofilia $\left(2.500 / \mathrm{mm}^{3}\right)$ y marcada trombocitosis que en 24 horas aumentó de 750.000 a un promedio de 1.500 .000 plaquetas $/ \mathrm{mm}^{3}$.

A una semana de la intervención presentó síndrome de respuesta inflamatoria sistémica (SRIS) con elevación de reactantes de fase aguda (VHS, PCR, procalcitonina y leucocitosis promedio de $20.000 / \mathrm{mm}^{3}$ ) y fiebre en patrón héctico. Se trató empíricamente con piperacilina sódica/tazobactam sódico más vancomicina y fluconazol completando 7 días. No se realizó esofagogastrograma de control. Se realizó TAC de tórax y abdomen con contraste que evidenció derrame pleural bilateral mayor a izquierda y ausencia de medio de contraste en vasos esplénicos pero bazo presente.

A dos semanas de la cirugía persistían parámetros clínicos y de laboratorio descritos agregándose sialorrea importante, dolor flanco izquierdo y polipnea. Se repitió TAC que mostraba persistencia del derrame, desaparición del bazo y reemplazo por gran colección intraabdominal de 16 x $18 \mathrm{~cm}$ con densidad de líquido-grasa-aire, múltiples burbujas en interior (Figura 1) y presencia de medio de contraste desde estómago hacia colección que se confirmó en esofagogastrograma.

Se instaló drenaje percutáneo Pig Tail 10F extrayéndose $400 \mathrm{~mL}$ de contenido hemático descompuesto confirmando infección por Streptococcus anginosus e indicándose tratamiento antibiótico con tigeciclina por 10 días, drenaje percutáneo diario y alimentación parenteral exclusiva.

Tras 25 días de tratamiento se observó respuesta favorable (remisión de síntomas, reinicio alimentación oral, desaparición derrame pleural y colección intraabdominal) (Figura 2) con cierre de comunicación gastroesplénica en esofagogastrograma de control (Figura 3), siendo dada de alta en buenas condiciones.

\section{Discusión}

A pesar de que sería fácil establecer a posteriori una relación de causalidad basada en los hechos presentados ("cierre" accidental de vasos esplénicos por clips, isquemia bazo, sobreinfección por fuga desde línea de sutura gástrica, gangrena

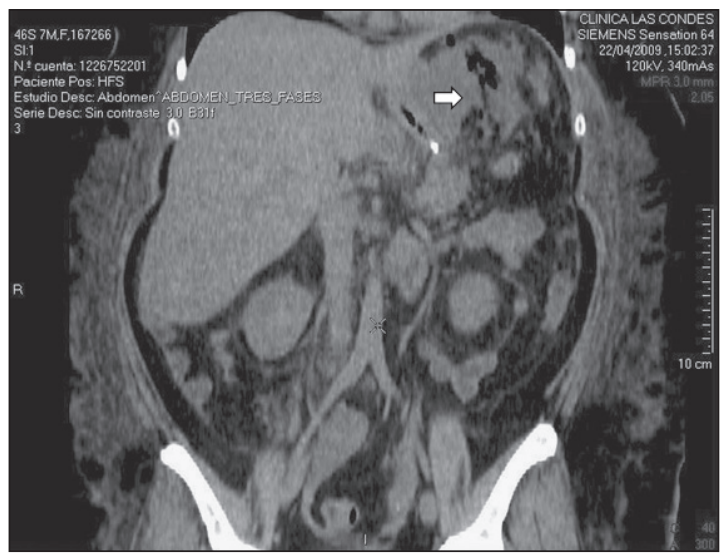

Figura 1. Colección intra abdominal (flecha) y comunicación gastroesplénica secundaria a gangrena esplénica.

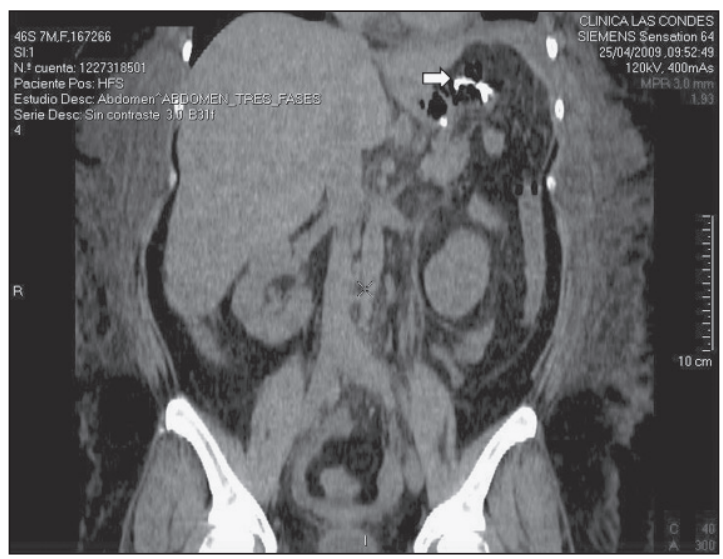

Figura 2. Colección intra abdominal (flecha) 3 días después de instalación drenaje percutáneo.

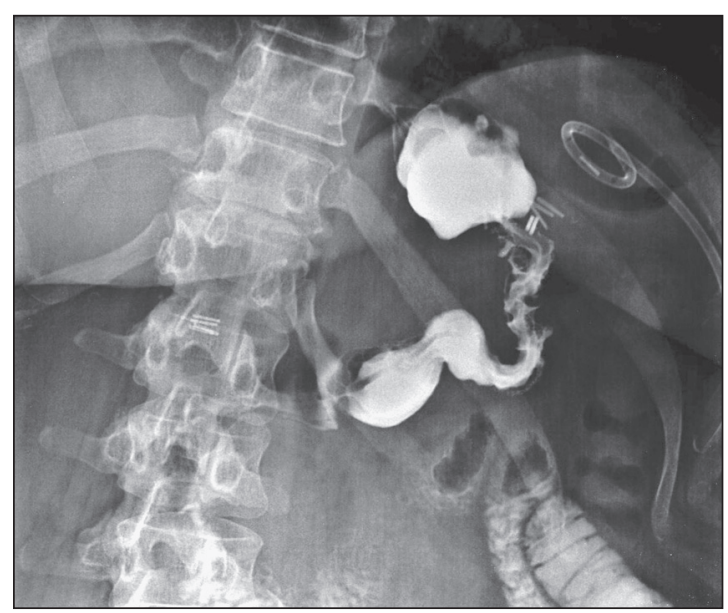

Figura 3. Comunicación gastroesplénica cerrada en esofagogastrograma de control. 
esplénica y comunicación entre ambos órganos), debemos destacar que inicialmente fue difícil el enfrentamiento del caso. Esto, dado que la presentación clínica descrita fue larvada y errática, además de la certeza señalada por los cirujanos tratantes, tanto de registro como verbal, de la nula sospecha de injuria esplénica, determinando así la evaluación por múltiples especialistas con diversas aproximaciones diagnósticas buscando otras causas que explicasen la clínica del paciente, ejemplo: atopia, parasitosis o reacción adversa a alguno de los fármacos administrados.

A pesar de ser una técnica relativamente nueva, dentro de las complicaciones que se mencionan en diversos estudios sobre manga gástrica laparoscópica, estos concuerdan en que su prevalencia es baja $(4,5-9,4 \%)^{18,1-20}$ y depende -teóricamente- de la habilidad quirúrgica, presencia de adherencias por cirugías previas y la necesidad de reintervención, factores por lo demás encontrados en este caso. En líneas generales, a corto plazo destacan como principales complicaciones la reintervención ${ }^{19}$, filtración de sitio operatorio ${ }^{19}$, fístula gástri$\mathrm{Ca}^{18} \mathrm{y}$ formación de abscesos ${ }^{20}$, no encontrándose reporte alguno de un completo infarto, infección y licuefacción del bazo así como su comunicación posterior con el remanente gástrico ${ }^{16}$. Por otro lado, sólo Fuks y cols ${ }^{18}$ señalaron una posible asociación entre la aparición de fístula gástrica y un IMC $>60$, pero no demostrado estadísticamente. Ninguno de los otros estudios establece riesgo relativo entre las complicaciones presentadas y otros factores como por ejemplo edad, comorbilidades asociadas o IMC al momento de la cirugía.

Con respecto a los microorganismos patógenos aislados, la gran mayoría de los abscesos presentaban cultivos polimicrobianos con predominio anaerobio y gram negativo, no encontrándose reportes de Streptococcus anginosus, el cual sólo ha sido aislado en los abscesos esplénicos de causa médica (ej. endocarditis bacteriana $)^{12,13}$ pues existe un predominio Gram positivos como Streptococcus sp $(65-83 \%)^{4,9,10}$.

Finalmente, y a modo de complementar el análisis de este tema en desarrollo, revisaremos las principales características de diagnóstico y tratamiento de los abscesos esplénicos cuyo manejo es independiente de si su etiología es médica o quirúrgica.

El bazo es un sitio poco común para la formación de abscesos $(0,2-0,7 \%)^{1-3,7,8,16}$, con una mortalidad que hoy en día es cercana a $0 \%{ }^{4,5,10}$, debido a su detección e intervención precoz.

Entre 61 y $64,7 \% 0^{2,4,9}$ de los abscesos esplénicos son solitarios, uniloculares, con diámetros de $1-18 \mathrm{~cm}$ y presentan mejor pronóstico ${ }^{2,4}$ pues son más sintomáticos y por ende detectables; por otro lado, los abscesos múltiples, símiles a la gangrena descrita, suponen $26,8-38 \% \%^{2,4,9}$ del total y son de peor pronóstico ${ }^{2,4}$, por ser menos sintomáticos y asociarse a sepsis independientemente del tiempo de resolución.

La cirugía, a pesar de representar traumatismo y riesgo de infección contigua, es factor de riesgo menor para desarrollar abscesos esplénicos, ubicándose tras causas médicas como embolia séptica, inmunosupresión y hemoglobinopatías ${ }^{1,4,5,9,13}$. Esto ha permitido describir condiciones de riesgo para desarrollar esta enfermedad (Tabla 1), en nuestro caso, serían las condiciones III y IV.

Clínicamente, la presentación puede ser variada (Tabla 2), no existiendo síntomas ni signos patognomónicos que diferencien contexto médico o quirúrgico, debiéndose tomar en cuenta entonces los antecedentes atingentes a cada caso. En este caso sólo se observaron los primeros tres hallazgos y nunca sialorrea que fue lo más molesto para la paciente.

Las distintas series, que incluyen etiologías médicas como quirúrgicas, señalan la leucocitosis como el parámetro de laboratorio más alterado $(79,6-100 \%)^{4,5,13}$ con promedio de 19.500 leucocitos $/ \mathrm{mm}^{3}$, lo cual también presentó la paciente. A destacar la trombocitosis rápidamente progresiva que permitió sospechar falla esplénica, hecho no reportado en otros trabajos consultados.

Los hemocultivos pueden ser negativos entre $11,4-28,7 \%$ y polimicrobianos en $10 \%$ de los

Tabla 1. Grupos de riesgo para desarrollar absceso esplénico

\begin{tabular}{|ll|}
\hline Grupo & Condiciones de riesgo \\
Grupo I & $\begin{array}{l}\text { Infección sistémica, paciente inmunocom- } \\
\text { prometido, bazo normal }\end{array}$ \\
\hline Grupo II & $\begin{array}{l}\text { Infección sistémica, enfermedad hematoló- } \\
\text { gica, esplenomegalia }\end{array}$ \\
\hline Grupo III & Trauma esplénico, daño en tejido esplénico \\
\hline Grupo IV & Infección directa, extensión por contigüidad \\
\hline
\end{tabular}

Nota: tomado y modificado con permiso de Chun et al ${ }^{1} \mathrm{y}$ Villamil-Cajoto 5 
Gangrena esplénica por Streptococcus anginosus - A. Rojas et al

Tabla 2. Síntomas y signos más frecuentes reportados en pacientes con absceso esplénico

\begin{tabular}{|c|c|c|c|c|}
\hline Manifestación clínica & $\begin{array}{l}\text { Chun C et al } \\
1900-1977^{1}\end{array}$ & $\begin{array}{c}\text { Nelken N et al } \\
1977-1986^{9}\end{array}$ & $\begin{array}{c}\text { Ooi L et al } \\
1987-1995^{2}\end{array}$ & $\begin{array}{c}\text { Yung et al } \\
1996-2005^{13}\end{array}$ \\
\hline Fiebre & 145/152 (95\%) & $131 / 156(84 \%)$ & 246/271 (91\%) & 20/21 (95\%) \\
\hline Dolor flanco izquierdo & $65 / 152(42 \%)$ & $64 / 152(42 \%)$ & $126 / 253(50 \%)$ & $8 / 21(35 \%)$ \\
\hline Esplenomegalia & $5 / 9(56 \%)$ & $82 / 152(54 \%)$ & 79/257 (31\%) & $6 / 21(29 \%)$ \\
\hline Derrame pleural izquierdo & $2 / 9(22 \%)$ & $34 / 173(20 \%)$ & $57 / 256(22 \%)$ & $2 / 21(10 \%)$ \\
\hline
\end{tabular}

Nota: tomado y modificado con permiso de Villamil-Cajoto ${ }^{5}$

casos $^{2,4}$. Son positivos en $70 \%$ de los pacientes con abscesos esplénicos múltiples pero sólo en $14 \%$ con abscesos únicos ${ }^{5,13}$. En este caso, los hemocultivos fueron siempre negativos y se aisló Streptococcus anginosus mediante cultivo directo.

El estudio imagenológico de esta paciente comenzó con una TAC de torax y abdomen con contraste cuya sensibilidad es cercana a $100 \%$, describiéndose áreas hipodensas con refuerzo periférico ${ }^{18}$, permitiendo el diagnóstico y facilitando el drenaje percutáneo. La ecografía abdominal, no solicitada, tiene sensibilidad de $75-93 \% 5,14$ debido a la interposición de la pared costal. En la radiografía de tórax pueden observarse derrame pleural, elevación hemidiafragma y atelectasias en $30-80 \%{ }^{4,5}$ por irritación subfrénica.

El tratamiento puede ser drenaje percutáneo o cirugía convencional. El primero puede utilizarse en pacientes hemodinámicamente estables, con abscesos únicos, sin tabiques internos y alejados del hilio esplénico. En este caso, la colección estaba en relación al hilio, presentaba tabiques internos y era de gran tamaño a diferencia de los drenados en otros trabajos cuyo tamaño no superaba los 10 $\mathrm{cm}^{14}$, lo que explicaría el por qué requirió más de 3 semanas de tratamiento. Gracias a radiólogos intervencionistas entrenados, las complicaciones y mortalidad son bajas y comparables a la cirugía en casos selecionados ${ }^{9,14}$. Como todo tratamiento con drenaje percutáneo, requiere una terapia antibiótica concomitante acorde a los microorganismos patógenos sospechosos señalados.

Revisiones desde 1980 a la fecha señalan la esplenectomía como el tratamiento estándar para los abscesos esplénicos -independientemente de su causa- pues permite eliminar rápidamente el foco infeccioso. Chun ${ }^{1,5}$ y Nelken ${ }^{5,9}$ recomiendan una intervención quirúrgica inmediata una vez hecho el diagnóstico; en cambio Ooi y $\operatorname{cols}^{2,5}$ sugieren que el drenaje percutáneo puede ser efectivo en casos seleccionados -como el presentado, sin compromiso hemodinámico y cuya cirugía conllevaba mayor riesgo de complicaciones inherentes-, sin embargo, debemos considerar que muchas veces se realiza en un paciente séptico pudiendo agravarla ${ }^{15}$. No obstante, en aquellos con recurrencia del absceso o mayor compromiso hemodinámico, no debe retrasarse la esplenectomía abierta ${ }^{15}$.

Es así como la gangrena y comunicación gastroesplénica corresponden a una complicación más de la manga gástrica laparoscópica y deben tenerse en cuenta en el postoperatorio de los pacientes sometidos a esta intervención, destacando finalmente la consideración que debe darse a la sospecha diagnóstica y a la hemostasia del sitio operatorio.

Agradecimientos: Quisiera agradecer a los médicos pertenecientes al Centro de Paciente Crítico y Unidad de Tratamiento Intermedio de la Clínica Las Condes por su apoyo en el desarrollo de este trabajo y por animarme a publicarlo.

\section{Referencias}

1. Chun C, Raff M, Contreras L, Varghese R, Waterman N, Daffner R. Splenic Abscess. Medicine (Baltimore) 1980; 59: 50-65.

2. Ooi L L, Leong S S. Splenic abscesses from 1987 to 1995. Am J Surg 1997; 174: 87-93.

3. Westh H, Reines E, Skibsted L. Splenic abscesess: a review of 20 cases. Scan J Infect Dis 1990; 22: 569-73.

4. Iñiguez A, Butte J, Zúñiga J, Torres J, Llanos O. Abscesos esplénicos. Comunicación de 7 Casos y Revisión Literatura. Rev Med Chile 2008; 136: 38-43.

5. Villamil-Cajoto I, Lado Fl, Van den Eynde-Collado A, Díaz-Peromingo J. Abscesos esplénicos. Presentación 9 
casos. Rev Chil Infect 2006; 23: 150-4.

6. Gadacz T, Way L, Dunphy J. Changing clinical spectrum of splenic abscess. Am J Surg 1974; 128: 182-7.

7. Bernabeu-Wittel M, Villanueva J L, Pachon J, Alarcón A, López-Cortés L F, Viciana P. Etiology, clinical features and outcome of splenic microabscesses in HIV infected patients with prolonged fever. Eur J Clin Microbiol Infect Dis 1999; 18: 324-9.

8. Hatley R M, Donaldson J S. Splenic microabscesses in the immunocompromised patient. J Pediatr Surg 1989; 24: 679-99.

9. Nelken N, Ignatius J, Skinner M, Christensen N. Changing clinical spectrum of splenic abscess. Am J Surg 1987; 154: 27-34

10. Lee C, Leu H, Hu T, Liu J. Splenic abscess in southern Taiwan. J Microbiol Inmunol Infect 2004; 37: 39-44.

11. Gatica M, Morales H, Espinoza A. Abscesos esplénicos múltiples, presentación de 2 casos. Rev Med Chile 1993; 121: 553-6.

12. Palavecino E. Streptococcus grupo anginosus: ies su identificación clínicamente importante?. Rev Chil Infect 2004; 21: 261-267.

13. Lombardi A, Coppola M, Pennica A, Maglio R, Moscaroli A. Splenic abscess due to Streptococcus anginosus. Case Report. G Chir 2008; 29 (8-9): 359-61.
14. Kogo H, Yoshida H, Mamada Y, Taniai N, Bando K, Mizuguchi Y, et al. Successful percutaneous ultrasoundguided drainage for treatment of a splenic abscess. J Nippon Med Sch 2001; 74: 257-60.

15. Farres H, Felsher J, Banbury M, Brody F. Management of Splenic Abscess in a Critically Ill Patient. Surg Laparosc Endosc Percutan Tech 2004; 14: 49-52.

16. Ballas K, Rafailidis S, Demertzidis C, Eugenidis N, Alatsakis M, Zafiriadou E, et al. Gastrosplenic fistula: A rare complication of splenic abscess. Surgical Practice 2005; 9: 153-5.

17. Yu J, Turner M, Cho S, Fulcher A, DeMaria E, Kellum J, at al. Normal Anatomy and Complications after Gastric Bypass Surgery: Helical CT Findings. Radiology 2004; 231: 753-60.

18. Fuks D, Verhaeghe P, Brehant O, Sabbagh C, Dumont F, Riboulot M, et al. Results of laparoscopic sleeve gastrectomy: a prospective study in 135 patients with morbid obesity. Surgery 2009; 145: 106-13.

19. Lalor PF, Tucker ON, Szomstein S, Rosenthal RJ. Complications after laparoscopic sleeve gastrectomy. Surg Obes Relat Dis 2008, 4: 33-8.

20. Frezza EE, Reddy S, Gee LL, Wachtel MS. Complications after sleeve gastrectomy for morbid obesity. Obes Surg 2009; 19: 684-7. 